\title{
ECOS DEL ENFRENTAMIENTO ARMADO GUATEMALTECO VEINTE AÑOS DESPUÉS DEL CONFLICTO. El ARRAIGO DE LA VIOLENCIA
}

\author{
Echoes of the Guatemalan Armed Confrontation Twenty Years after the Conflict. \\ The Persistence of Violence
}

\author{
David Caballero-Mariscal
}

Resumen: El 29 de diciembre de 1996 marcó un hito en la historia reciente de Guatemala al suscribirse los acuerdos de paz que pusieron fin a un enfrentamiento de 36 años. Veinte años después la violencia continúa presente en el país. En el artículo se describe la situación actual de la violencia en Guatemala como consecuencia de lo experimentado en el periodo de enfrentamiento. Se utiliza una metodología descriptiva, ilustrada con datos cuantitativos procedentes de diversas fuentes y con el análisis de una encuesta cerrada de elaboración propia. Se concluye que el periodo posconflicto supone la continuación de un paradigma similar de violencia.

Palabras clave: paz conflicto armado, reconstrucción, violencia.

Abstract: Peace Agreements were signed in Guatemala on December 29, 1996. This was a significant milestone in the country's recent history, insofar as they held out hope that the 36-year-old conflict would end, as would human rights violations and ethno-genocide. Notwithstanding the commemoration of the 20th anniversary of the signing of the Accords, violence continues to be at the forefront in the Land of Eternal Spring. The root causes of the current situation probably lie in the armed confrontation. The focus of this paper is to describe the well-embedded, ongoing violence in Guatemala, as a consequence of the previous period. To this end, we use a descriptive methodology to undertake a thorough inquiry, with data from various sources and from an analysis of a questionnaire prepared by the author. I conclude that the current post-conflict period will involve continuity of this criminal paradigm.

Keywords: peace, armed conflict, reconstruction, violence.

David Caballero Mariscal. Doctor en Literatura por la Universidad de Granada y doctor en Antropología por la Universidad de Huelva, España. Profesor-investigador en el Departamento de Humanidades y Ciencias Sociales, Centro de Magisterio La Inmaculada, de la Universidad de Granada, España. Temas de especialización: paz, conflictos, antropología aplicada, educación. Correo electrónico: davidcaballero@ugr.es.
Enviado a dictamen: 12 de enero de 2017. Aprobación: 22 de septiembre de 2017. Revisiones: 2. 
L a bisagra del cambio de milenio, que se auguraba como un umbral de profundas trasformaciones, las cuales se han visto limitadas por elementos históricos muy significativos a nivel global, ha sido fundamental en Guatemala en muy diversos aspectos. Por un lado, tras un duro enfrentamiento armado que se prolongó durante más de tres décadas y que dejó más de doscientos mil muertos (Duque, 2007), cuarenta mil desaparecidos y casi cien mil desplazados (González Sanz, 2014), y después de que la intensidad de la represión alcanzara el punto máximo de violencia en el denominado "quinquenio negro" (García, 2005), un periodo de paz de "baja intensidad" (Gutiérrez, 1998) sentó las bases para la firma de acuerdos entre las partes en diciembre de 1996.

La realidad muestra que la violencia es una característica de la región centroamericana, en especial en el denominado "triángulo norte" - Guatemala, Honduras y El Salvador-, y es complejo determinar las causas que llevaron a esta situación, que se percibe en la actualidad como irreversible. En este sentido, Cruz sitúa al Estado mismo, por medio de sus instrumentos y mecanismos, como causante y responsable directo de parte de la violencia y la criminalidad reinantes (Cruz, 2010: 226). Por otro lado, la pobreza y la desigualdad se convierten en factores relevantes para comprender el impacto de la exclusión socioeconómica, y en causantes fundamentales de la violencia criminal. Si se considera el coeficiente Gini para medir el índice de desigualdad en Guatemala, se halla que, a nivel mundial, esta nación centroamericana se encuentra en novena posición, con un índice de 52.4, tan sólo superado en la región por Honduras. Si bien en un lustro ha bajado más de un punto, ya que en 2011 se situaba alrededor del 53,7 (UNDP, 2011 y 2016), el desarrollo económico del país continúa siendo muy discreto (0.56).

Durante el conflicto, no todo el país se vio afectado de la misma manera. Así, de acuerdo con la Comisión de Esclarecimiento Histórico (CEH, 1999), la Oficina de Derechos Humanos del Arzobispado de Guatemala (ODHAG, 1998) y Aguirre Tobón (2014), los departamentos más afectados fueron Quiché, Huehuetenango, Alta Verapaz, Chimaltenango y Baja
Verapaz, además de la ciudad capital. La represión hizo que estas zonas fueran más susceptibles a ciertas formas de exclusión, discriminación y trauma colectivo, si bien en la actualidad los núcleos más relacionados con los delitos y la criminalidad son los urbanos, en especial la ciudad capital, su área metropolitana y otros núcleos grandes de población. No obstante, la espiral de la violencia alcanza, aunque no en la misma medida, a toda la geografía nacional.

Asistimos en la actualidad a un cambio de paradigma que ha provocado movimientos masivos de índole multicausal, pero que refieren, en palabras de Varela Huerta, a una "trinidad perversa" compuesta básicamente por la violencia del mercado, del Estado y la patriarcal (Varela, 2016: 19). De fondo se encuentran la impunidad, el poder de los cárteles y, sin dejar de ser desdeñables, las temidas "maras", que desde una compleja composición y génesis han ido alzándose en el panorama de la inseguridad. Aunque Villa, Londoño y Barrera subrayan la relevancia de "la reconstrucción del tejido social, en la generación de procesos de reconciliación y la transformación del sujeto individual y colectivo" (2015: 217), esta cuestión resulta relativa cuando el cambio de una sociedad bélica a otra posbélica sólo se percibe por la firma de unos acuerdos y la consideración interna y externa de no estar en período de conflicto.

El objetivo fundamental del presente artículo es analizar la situación del país en el período posconflicto, así como describir la percepción actual que tiene la sociedad guatemalteca sobre la violencia dos décadas después de la firma de los acuerdos de paz. Se parte de la hipótesis de que la violencia está arraigada en Guatemala y se intenta mostrar cómo existe una conexión entre el proceso bélico de 36 años y la actual perseverancia de la violencia, como elemento estructural, a modo de gran trauma colectivo heredado. Los agentes directos e indirectos que causaron la violencia en el pasado dejaron su impronta en la sociedad de manera tan sólida, que ésta en ocasiones se percibe con naturalidad.

Mediante la metodología cualitativa acompañada de datos cuantitativos, se procedió a efectuar un análisis de la situación en el país, de la perspectiva de la violencia 
como heredera de los horrores del enfrentamiento, y de la percepción que tenían los ciudadanos sobre la realidad que se vivía en Guatemala en 2016, dos décadas después del final del conflicto. Para ello se revisaron datos significativos procedentes de fuentes estadísticas fiables para establecer una comparativa acertada. Posteriormente se procedió a entrevistar, mediante un cuestionario de elaboración personal, a población guatemalteca de diversos lugares del país, con una selección aleatoria de la muestra, pero representativa. Por el temor generalizado, se percibieron dificultades en la participación de los entrevistados, entre quienes se encontraban colaboradores y conocidos a los que se contactó siguiendo la técnica "bola de nieve" descrita por Cea d'Ancona (2001).

\section{Transformación y continuidad de la violencia}

\section{Factores políticos $y$ sociales}

Se puede considerar, dos décadas después del enfrentamiento, que existe una conexión entre la violencia del conflicto y la existente en el periodo posterior a la firma de los acuerdos de paz. En este sentido, resulta trascendente matizar algunos aspectos. Kalyvas (2006) afirma que la distinción entre un enfrentamiento armado o guerra civil y el posconflicto se sitúa en el monopolio de la violencia por parte del Estado. Así, el aparato central de poder y los mecanismos licitados de manera encubiertamente oficialista marcarían la distinción entre los grupos disidentes, mientras que las formas y la intensidad de la violencia los harían converger. Desde esta perspectiva, el fin del conflicto no implica necesariamente el fin de la violencia, como ocurre en el caso de Guatemala y en los de otras naciones que han experimentado procesos análogos.

McNeish y López (2012) indican que la guerra experimentada por décadas en Guatemala no es sólo un recuerdo en el país, sino que sus formas perseveran, por lo que los acuerdos de paz implicaron un hito y un logro, pero no pusieron fin sino a determinados aspectos de la convivencia, a veces exclusivamente formales.
Es evidente que los años de duros enfrentamientos y violaciones de los derechos humanos se hallan intrínsecamente conectados con la violencia en la actualidad. Como subrayan Kurtenbach y Wulf (2012), la frágil situación posconflicto en muchos contextos en los que la guerra fue intensa, mantenida en el poder y bajo la influencia de potentes aparatos de poder, ha favorecido la perseverancia de escenarios violentos.

Los grupos de contrainsurgentes que actuaron de forma clandestina, ligados de una manera u otra a los aparatos de poder, dejaron una impronta en métodos silenciosos de actuación y en prácticas como la impunidad. De igual manera, se detecta una continuidad muy marcada entre los métodos brutales empleados durante el enfrentamiento y las formas desgarradoras de violencia actuales. Como subraya Lambach, Guatemala pertenece a un grupo de sociedades posconflicto de tipo II, es decir, aquellas en las que los conflictos se caracterizaron por la alta intensidad y, al mismo tiempo, por la ausencia de intervención de agentes externos. Por ello, tras la época de guerra, "la coalición ganadora pudo haberse fragmentado en el entorno posconflicto, direccionando hacia la reconstitución del oligopolio, aunque con distintos actores" (Lambach, 2007: 12, traducción propia).

Destaca un elemento que no se puede pasar por alto y al que debe aludirse de forma precisa: la desmilitarización. Ésta constituye uno de los aspectos más destacables en relación con los factores que han determinado la transición entre la guerra y la posguerra violenta, y vino acompañada de la inercia de la militarización heredada. De hecho, Zartman expresa que la consecución de la paz no implicaría en sí "un arreglo definitivo de problemas específicos (por prominentes que puedan ser determinadas injusticias entre las causas del conflicto), sino más bien una restauración de la política normal" (Zartman, 1995: 24).

En Guatemala, la transición se llevó a término con la intervención de todos los agentes implicados en el enfrentamiento. Además, el primer gobierno de la paz, presidido por Álvaro Arzú, a pesar de ser de corte conservadurista liberal y de ideología derechista, abrió la perspectiva del desarrollo social y se distinguió 
sobremanera de los gobiernos militares anteriores caracterizados por políticas represoras (Pásara, 2003). No obstante, bajo el gobierno de Portillo, su sucesor en el cargo, se vislumbraba, además de la sombra de uno de los grandes genocidas - Ríos Montt-, el peso de la corrupción, que también persiguió a posteriores gobiernos liberales que alternaron con hijos del militarismo.

Son tres los ejes que deben considerarse. El primero de ellos se basa en el arraigo de las estructuras militares, que están aún presentes y arrastran consigo dinámicas de represión social, aunque se haya trabajado en su disolución deforma progresiva. A principios del presente milenio, y durante toda esa década, se celebraron en Guatemala los logros de la desmilitarización, si bien ésta no se pudo alcanzar de forma total. El segundo eje procede de la perspectiva social de la militarización en las estructuras poblacionales que, condicionadas por sus relaciones sociales y comunitarias, han vivido, transmitido o adquirido estas inercias de manera natural por aprendizaje vicario. El tercer eje se basa en quienes abandonaron poderes militares, paramilitares y guerrillas, que pasaron a trabajar en la seguridad privada debido a la relevancia que adquirió esta actividad (Saavedra, 2014) por la ausencia de un sistema legal eficiente. Por otro lado, tanto miembros procedentes de los sectores armados durante el enfrentamiento, como personas de nuevas generaciones, pasaron a formar parte de grupos del crimen organizado y fuerzas afines. Algunos grupos se hallan vinculados al narcotráfico, mientras otros, de una forma más o menos directa, han sido utilizados por los gobiernos de transición y de la época de la paz "consolidada" para poner en práctica políticas de terror, limpieza social y mantenimiento del orden. De ahí que Peacock y Beltrán hablen de hidden powers (2003: 1), grupos clandestinos ilegales que han actuado con respaldo estatal o bajo intereses diversos, pero de forma clandestina.

\section{Impunidad como eje de la violencia}

Una de las principales causas para comprender la ola de violencia en Guatemala encuentra su génesis principal en la impunidad. Ésta se puede traducir comola ausencia radical de respeto a los derechos humanos en tanto que se producen acciones criminales arbitrarias de las que los autores salen incólumes. El valor de la vida es bajo en proporción a lo costoso que resulta la investigación y el hallazgo y procesamiento de criminales (International Crisis Group, 2010). El efecto más inmediato de esta circunstancia es una desconfianza significativa en la justicia, que exculpa a los verdaderos responsables e incita a que los actos criminales se repitan (Cabrera, 2001). Así, las verdaderas raíces de la problemática no han sido atajadas y se procede a una solución de los síntomas sin tratar la verdadera patología.

La génesis de esta impunidad, que aún hoy día se proyecta, se encuentra en la época del enfrentamiento armado. Las políticas cruentas de los gobiernos represores, en especial a lo largo del denominado "quinquenio negro" (1978-1983), en el que se produjeron el 90\% de las violaciones de los derechos humanos (Cuesta, 2001; Bastos y Camus, 2003; García, 2005), generalizaron métodos de una eficacia radical debido a su crueldad. Los "escuadrones de la muerte", también presentes en otras naciones de Latinoamérica, sembraron el terror (Szichman, 2013), y el punto más radical llegó de la mano de las denominadas políticas de "tierra arrasada". Estas políticas, junto a otras contrainsurgentes, siguen proyectando un eco significativo, puesto que muchos de los que las llevaron a término continúan impunes dos décadas más tarde, y más de treinta años después de haber sido acometidas (García Carrera, 2015).

Dos elementos pueden considerarse como el reflejo más significativo de la impunidad. El primero lo constituye el asesinato de monseñor Gerardi, principal promotor de la memoria histórica y coordinador del informe Guatemala nunca más (ODHAG, 1998), pocos meses después de la firma de los acuerdos de paz, el 23 de abril de 1998. Todavía hoy no se han esclarecido del todo las circunstancias de su asesinato $\mathrm{y}$, a pesar de que existen tres imputados por el crimen, el caso continúa siendo un espectáculo mediático del que han salido victoriosos los verdaderos responsables (González, 2007). El otro elemento vertebrador de la 
impunidad se encuentra representado en la figura de dos grandes genocidas: Ríos Montt y Romeo Lucas. Como se ha mencionado, durante sus mandatos, en el "quinquenio negro", se cometió la mayor parte de las violaciones de los derechos humanos. Sus periodos de gobierno fueron breves, en especial el de Ríos Montt, pero este hecho no impidió que puedan considerarse como los más represivos. En el caso de Romeo Lucas García, éste falleció en Venezuela en 2006 sin asumir las consecuencias por sus actos, a pesar de la represión que emprendió contra los intelectuales, las múltiples masacres que se llevaron a cabo durante su mandato y el proceso que tiene abierto por la quema de la embajada de España en 1980. Por su parte, Ríos Montt ha sido procesado en diferentes ocasiones por genocidio y crímenes de lesa humanidad, pero de todas ellas ha salido impune. A pesar de los pasos que se han dado para proceder al fin de la injusticia, y de "la carga simbólica que significa para las víctimas el hacer justicia en un país históricamente marcado por la impunidad" (Rodríguez y Gutiérrez, 2015: 57), parece que el fin de ésta aún no termina de alcanzarse. Así, el 10 de mayo de 2013 se alcanzó un auténtico hito contra la impunidad, porque por primera vez las instancias de impartición de justicia dieron el paso de hallar culpable a Ríos Montt por delitos de genocidio durante su mandato presidencial entre 1982 y 1983, aunque una decisión posterior anuló esta sentencia (Benítez, 2015).

\section{Las formas de la violencia. La muerte como realidad}

Además de constituir el exponente máximo de la cultura de la violencia y de suponer en sí mismas una prolongación del conflicto armado, estas formas de terror social a las que nos referíamos previamente, llevadas a término fundamentalmente por los grandes genocidas del país, son reflejo de una idiosincrasia adquirida dramáticamente y de una continua tensión generalizada. Las grandes desigualdades reinantes se alzan con el monopolio de la raíz de esta situación de impunidad, represión y violencia generalizadas en los años más oscuros del genocidio (González,
2007). Aunque la violencia alcanza a cualquier sector de la población, hay colectivos susceptibles de mayor vulnerabilidad, entre ellos, la población indígena, las mujeres y la infancia (ver gráfica l y tabla l).

Si se consideran los datos plasmados en la tabla 1, podrá llegarse a varias conclusiones. La primera de ellas es que la transición hacia la paz no se ha visto reflejada en una reducción de víctimas; por el contrario, tras la firma de los acuerdos de paz el número de víctimas aumentó año tras año hasta alcanzar su punto culminante en 2008, con un repunte en 2014, uno de los años más cruentos.

Otra cuestión a tener en consideración es la discrepancia entre los datos de las diversas fuentes. Si bien éstos coinciden hasta 2011, a partir de este año se observan diferencias considerables. Por un lado, en las fuentes policiales sólo figura un recuento de las víctimas que han sido sometidas a análisis o a autopsia, aunque en algunos casos no se ha logrado su identificación, mientras el Grupo de Apoyo Mutuo (GAM) considera otras fuentes e incluye víctimas que no han sido analizadas por la policía ni incluidas en las fuentes estadísticas oficiales.

El año 2016 terminó con un índice de criminalidad ligeramente inferior al año anterior, aunque los primeros meses fueron especialmente duros por la crueldad de los actos que se produjeron, como desmembramientos.

La lentitud de los procedimientos judiciales refleja la ineficacia del sistema legal. Este hecho, unido a la impunidad, la corrupción policial y otros factores asociados, conlleva la desconfianza en los cauces judiciales. Las bases de esta situación generalizada se sentaron durante la época de guerra por la actuación de los diferentes gobiernos y sus brazos armados; posteriormente, ciertas políticas de exterminio basadas en la limpieza social (Ramírez, 2000: 147) no sólo contradicen la consecución de la misma paz, sino que además inhiben el camino hacia la verdadera democratización y resucitan la avidez por la vuelta a los regímenes represivo-autoritarios (Fernández Rupérez, 2007). Esta circunstancia no sólo frena el crecimiento económico de la nación, sino que repele las inversiones de capitales extranjeros y el auge del turismo, además de 
que favorece la salida del país de una oleada de "nuevos refugiados" que huyen de conflictos encubiertos. Y es que, tal y como subraya Gómez Johnson, en toda la región centroamericana y en México se ha pasado de una situación de migración por motivos económicos a "la migración forzada por efecto de la violencia" (2015: 199), lo que ha conducido al surgimiento de una modalidad de refugiados que no existía dos décadas atrás, pero que refleja una situación análoga a la experimentada durante el enfrentamiento.

La Organización Mundial de la Salud considera que una tasa de homicidios de 10 implica un grave problema de salud pública a nivel epidémico Si se considera que en los 36 años de enfrentamiento se producía una media de diez víctimas diarias, veinte años después, muy a pesar de los acuerdos de paz, esta cantidad se duplica.

\section{Las mujeres como primeras víctimas de la violencia}

Un hecho bastante generalizado a nivel global es la asimetría de derechos entre mujeres y hombres. En el caso específico de Latinoamérica, son muchas las razones que pueden aducirse para subrayar que la lucha por la equidad es una constante, pero a menudo está cargada de limitaciones y dificultades. Los factores sociales, el peso de la tradición y otros elementos han llevado a una situación límite a las mujeres, quienes padecieron durante el proceso bélico un recorte en sus derechos y un rebajamiento de su dignidad. Así, la Defensoría de la Mujer Indígena destaca que, en las múltiples violaciones de los derechos humanos cometidas en los márgenes del conflicto, las mujeres mayas se llevaron la peor parte puesto que, además, fueron a menudo consideradas como auténticos botines de guerra (DEMI, 2007).

La violencia contra las mujeres adquiere muy distintas formas, de las cuales la más grave es el feminicidio. Guatemala ostenta la tercera posición en número de asesinatos de mujeres en Latinoamérica, superada sólo por Jamaica y El Salvador. Con una tasa de feminicidios del 9.1 y un número total de muertes de 846 durante el año 2014, se puede afirmar que la situación continúa siendo de auténtico drama. En 2015, el
Instituto Nacional de Ciencias Forenses de Guatemala reportó 563 casos de asesinatos de mujeres de forma violenta (Kristinsdóttir, 2015). Si bien esta cantidad supone una disminución considerable respecto del año anterior, desde 2005 hasta 2014 la cifra anual de muertes violentas de mujeres nunca fue menor a seiscientos casos. Si se analiza el total de feminicidios reportados entre 2000 y 2015, se acumularon alrededor de nueve mil víctimas (INACIF, 2015; GAM, 2015a), aunque el número real fue superior por la amplia incidencia de la violencia intrafamiliar y casos no reportados.

Además de lo anterior, la mutilación, la tortura y el ensañamiento son habituales. Se debe subrayar que en la última década han proliferado los tribunales especializados en la violencia contra las mujeres, y ante las múltiples denuncias por abusos y malos tratos, que pueden contabilizarse por millares, durante 2015 se dictaron 1517 sentencias, un centenar más que el año anterior y un $1000 \%$ más que una década antes (OACNUDH, 2016: 11-12).

Adicionalmente, se producen otras formas de violencia muy destacadas contra las mujeres que incluyen maltratos tanto físicos como psicológicos, violación y falta de acceso a los mismos derechos que los hombres —educación, sanidad, nutrición-. Otra cuestión importante es la triple discriminación en la que se encuentran inmersas las mujeres: por género, por origen étnico — maya - y por nivel social (Valdez, 2016).

En lo que se refiere a alfabetización, si bien en las dos décadas transcurridas desde el fin del conflicto el porcentaje de analfabetismo ha pasado de acercarse al $40 \%$ a bajar al $15 \%$, entre las mujeres indígenas del ámbito rural, siguiendo datos del INE-ENCOVI y la ONU (2015/2016), el analfabetismo supera el 20\%, si bien se halla en retroceso.

Se ha de subrayar que sólo entre uno y cuatro casos de cada cien de violaciones, agresiones y asesinatos de mujeres han llegado a ser procesados judicialmente hasta 2016, por lo que los ecos de la justicia en Guatemala se hallan aún más inhibidos en el caso de las mujeres (Eguizábal, 2015). Los más de 9700 feminicidios ocurridos en los últimos quince años dan testimonio de este hecho. 


\section{La vulnerabilidad de la infancia ante la violencia}

No hay duda de que en una sociedad frágil, caracterizada por la inestabilidad, uno de los sectores más vulnerables y susceptibles de padecer explotación y de que no se respeten sus derechos es la infancia. Ante la pobreza y la necesidad de subsistir, los menores se convierten en un apoyo para contribuir a los ingresos familiares.

Para comprender la situación de la infancia, es importante conocer las tasas de mortalidad infantil y su proceso de evolución en los últimos años (ver tabla 2). En este sentido, la realidad muestra un descenso considerable de la tasa de mortalidad infantil en el periodo posconflicto, aunque prevalecen patologías y enfermedades que causan muerte que tienen su origen en la falta de recursos, una nutrición inadecuada y la ausencia de acceso a servicios de salud. Estas condiciones se ven agravadas en las comunidades rurales más aisladas, en las que aún resulta más dificultoso el acceso a diferentes recursos. Otro factor de riesgo para los menores lo constituye su exposición a trabajos no propios para su edad. En este sentido, se estima que casi un millón de niños de entre siete y catorce años trabaja habitualmente. Es cierto que la tasa de población infantil sometida a trabajos se ha reducido ligeramente, puesto que en 2010 alcanzaba el 66.8\% en las áreas rurales, y en la actualidad, según datos de UNICEF (2016) se reduciría en más de diez puntos. Por otro lado, se tiene información de que unos 12000 menores de entre cinco y seis años dedican más de cuarenta horas de trabajo a la semana (Maldonado, 2009; INE, 2016).

Una de las cuestiones más preocupantes a propósito de la infancia es la gran cantidad de niños que viven en la calle, cuyo número ha ido en aumento en la última década. Si en 2007 la Oficina de Derechos Humanos del Arzobispado de Guatemala situaba en 3200 el número de menores sin hogar, para principios de 2016 la cifra parecía superar los 5300, con todo lo que este hecho conlleva en cuanto a la susceptibilidad de abusos, asesinatos, tráfico de personas y explotación.

Si se consideran las estimaciones de UNICEF (2010), puede comprobarse que casi la mitad de la población infantil, en especial la proveniente de ámbitos rurales e indígenas, había padecido en la primera década del milenio desnutrición en algún grado. Esta situación, según las estimaciones del gobierno, cambió considerablemente en los primeros quince años del siglo XXI (OMS y OPS, 2016), aunque en este período se han producido varias crisis de desnutrición infantil en distintos departamentos.

\section{Violencia colectiva}

Entre finales de la década de los setenta y mediados de los ochenta, los distintos grupos militares y afines sembraron el terror de manera generalizada, sobre todo entre la población maya. Si bien ningún sector se vio libre de las consecuencias de la violencia, las comunidades rurales padecieron los mayores actos de violación de los derechos humanos (ODHAG, 1998; CEH, 1999; Sichar, 2001). La violencia engendró una espiral de violencia aún mayor; la crueldad de las formas y la obligación de contemplar torturas y violaciones de los derechos humanos por parte de los familiares y vecinos de las comunidades como medida ejemplarizante dejó una huella significativa en las comunidades. En casos más extremos incluso se llegó a obligar a miembros de familias y comunidades a perpetrar tales actos. Como consecuencia, a lo largo de las décadas en la colectividad y su inconsciente parece haber quedado impresa la impronta de esta violencia de carácter colectivo. Los "escuadrones de la muerte", los "pelotones de la muerte" (Vela, 2014) y otros sistemas especiales impulsados por los gobiernos represivos fueron reemplazados por las turbas enfurecidas ante los continuos robos, extorsiones, asesinatos y violaciones. De fondo se observa una repetición de esquemas similares: sin mediación de palabras ni aplicación de leyes, se procede a ejecuciones extrajudiciales violentas y crueles.

Existe un paralelismo entre las masacres cometidas durante el enfrentamiento y los linchamientos que han tenido lugar a partir de la firma de los acuerdos de paz en 1996 fundamentado en tres ejes principales: la ejecución de forma violenta por parte de un grupo hacia 
una o varias víctimas, la extrema violencia en las formas, y la participación indirecta, a modo de espectadores, por parte de todo el grupo. Lo único que discrepa en ambos casos es el carácter articulado y premeditado del acontecimiento en sí y la motivación de la participación por parte del grupo, que en el conflicto funcionaba como medio de represión y eliminación de los sectores que pretendían ser suprimidos, mientras que en el caso de los linchamientos se llevan a cabo por intentos colectivos de impartir justicia. Este hecho no exime de la actuación descontrolada, impulsiva y extremadamente violenta (Vilas, 2005) (ver gráfica 2).

La violencia colectiva surge como respuesta de la población a los asaltos, extorsiones y falta de justicia. Diversas asociaciones se han encargado de tratar de crear conciencia en las comunidades, e incluso de intervenir ante la sospecha de linchamientos, pero éstos siguen siendo frecuentes, como se puede comprobar en la cifra correspondiente a 2016: 31 víctimas mortales durante sólo el primer trimestre (GAM, 2016).

En cuanto al fenómeno del linchamiento, éste se puede concebir como un acto compartido de violencia hacia un individuo o varios, que a priori tiene la intencionalidad inherente de aplicar justicia, con o sin la finalidad de aniquilar. En cualquier caso, destaca la fuerte agresividad empleada y las nefastas consecuencias, así como la participación del grupo en la ejecución, o su pasividad ante el acontecimiento por contemplación y omisión (MINUGUA, 2001).

En la situación de etnocentrismo que se observa en Guatemala, con una escisión social evidente entre indígenas y ladinos, y donde estos últimos ostentan el monopolio del poder económico y social, se ha tratado de buscar causas a este comportamiento de violencia colectiva en la propia idiosincrasia indígena. Se trata de planteamientos a todas luces fundados en tal perspectiva etnocéntrica (Casaús, 2002), y que Ixcol considera que "están configurados bajo la visión dominante o hegemónica que los utiliza para denostar y criminalizar las prácticas culturales indígenas y mayas", ya que "en la cultura maya no se maneja ni la noción de linchamiento (inexistente en el idioma) ni la comprensión occidental del mismo" (Ixcol, 2013:
4). Por lo anterior, puede considerarse que esta forma de violencia colectiva responde al hastío por la falta de justicia y el reinado de la impunidad. Por otro lado, existe una conexión entre los lugares en los que se produjeron masacres profundamente violentas y aquellos en los que se han producido linchamientos con desenlace fatal y la utilización de métodos muy duros (Mendoza, 2003). A este respecto, Fernández García (2004) se refiere a la "semilla" y el "fruto" para tratar de explicar en parte los hechos de violencia colectiva en forma de linchamientos.

\section{Pandillas y maras. Terror social por el crimen organizado}

La violencia colectiva posee tres niveles de expresión en la región de Centroamérica y, concretamente, en Guatemala. El primero de ellos, de carácter estructural, se corresponde con el arraigo de la violencia, de tal forma que ésta se halla integrada de forma tan inherente a la realidad, que forma parte de la vida cotidiana. El segundo nivel se corresponde con una forma de ajusticiamiento poco organizado y nada estructurado del que nos hemos hecho eco con anterioridad, y que se traduce en los linchamientos. El tercer nivel, uno de los más significativos y problemáticos, sí tendría un carácter estructurado y se hallaría en relación con el narcotráfico y el crimen organizado.

Kurtenbach (2008) señala que la juventud es el auténtico chivo expiatorio, motivado por las fracturas generadas de la época posconflicto. Así, si durante la época más álgida del enfrentamiento el foco de atención se centró en los grupos guerrilleros y las políticas de terror, la cuestión ha cambiado sobremanera al ser la violencia organizada uno de los mayores problemas de la nación. Esta realidad ejerce su influencia más directa sobre los grupos de población más jóvenes.

Aunque es difícil determinar el origen de las maras y de las pandillas centroamericanas, es una realidad constatable que existe una relación entre el crimen organizado y la realidad experimentada en la época del conflicto. En su génesis, además de la relación con los respectivos conflictos décadas atrás, las maras derivan de la emigración de centroamericanos, en especial de 
salvadoreños, a ciudades de Estados Unidos como Los Ángeles. Allí, ante la exclusión social y como mecanismo para sobrevivir y proteger sus intereses, traducidos a menudo en clave territorial, se organizaron en pandillas cuyas formas de enfrentamiento se tornaron cada vez más violentas. Tras la deportación masiva de miembros de estos grupos, al llegar a los países de sus raíces encontraron estructuras propicias para la organización del crimen y la aplicación de los esquemas adquiridos en origen (POLJUVE, 2007). La fragilidad de la paz y la cultura de la violencia heredados de los fuertes conflictos internos generaron el caldo de cultivo propio para que las maras arraigaran de una forma eficaz.

Tradicionalmente, las dos maras que más relevancia han tenido son la 18 y la Salvatrucha. La primera debe su nombre a la calle en la que surgió en la ciudad de Los Ángeles, y la segunda apareció para diferenciarse cultural e idiosincráticamente de la anterior. Las maras no constituyen un fenómeno que se delimite a un simple grupo u organización, sino que sus alcances son mayores no sólo porque la violencia ejercida por estos grupos se acerque al 20\% de los actos delictivos y asesinatos acontecidos en Guatemala durante los últimos veinte años (Banco Mundial, 2010; Sánchez, Scott y López, 2016), sino, además, porque las pandillas en sí sólo son la punta del iceberg de una trama estructural mucho mayor y más compleja. De esta forma, y siguiendo a Cardenal (2008), las pirámides de las maras se apoyan en esquineros que no pertenecen a las organizaciones, pero que se hallan cercanos a éstas y se encuentran en parte a su servicio como observadores o participantes de pequeños hechos delictivos. Al no cumplir con ciertos requisitos, como haber superado la terrible "clica" o prueba inicial de aceptación, no forman parte directa del grupo, pero contribuyen a las acciones violentas y son un elemento esencial en ellas. Un nivel superior viene de la mano de las "pandillas" de barrio, de las que las maras se nutren, y por encima se encuentran no sólo las maras en sí mismas, la Salvatrucha y la 18, sino que desde hace más de una década, y en directa relación con el narcotráfico mexicano, participan otros grupos como los Zetas, además de otros de origen insular como los Neta (Zuzunaga, 2011).
En un nivel superior se halla una complicada red de células grupales entretejidas en la actividad del crimen organizado y el tráfico de drogas, armas y personas, de las que forman parte sicarios, antiguos militares y afines. Si bien han cambiado las formas, las élites del poder y los intereses, la política de terror y el miedo continúan presentes en la sociedad.

Se han llevado a cabo políticas determinadas para erradicar las maras, sobre todo las denominadas "de mano dura", la "limpieza social" y los intentos de reimplantación de la pena de muerte. La aplicación de estas medidas, marcadas por la violencia, tiene en común con las políticas de limpieza social llevadas a cabo durante el genocidio el hecho de fundarse en relaciones asimétricas de poder. Se percibe un eco directo que conecta con la realidad del conflicto, con estructuras similares y sentimientos sociales paralelos.

\section{Percepción de la situación actual de la problemática en el país}

Tras realizar un análisis de la bibliografía existente y cotejar de manera contrastiva las fuentes estadísticas, descriptivas, históricas y actuales, se procedió a administrar una encuesta de elaboración propia, con motivo del cumplimiento de los veinte años de la firma de los Acuerdos de Paz. Para ello, se llevó a término, como punto de partida, un sondeo de opinión acerca de si hubo genocidio en el país, para posteriormente analizar la percepción sobre la violencia en Guatemala. Se seleccionó una muestra de $\mathrm{N}=373$, para la primera parte de la encuesta, y de $\mathrm{N}=565$ en las sucesivas. La población se escogió de entre diversos municipios en los departamentos de Alta y Baja Verapaz, Esquipulas y Guatemala capital. Se empleó la metodología cuantitativa para ilustrar los datos, usándose un cuestionario cerrado, de elaboración propia y fundado en las problemáticas que, por medio del trabajo de campo, se fueron observando en las campañas etnográficas. El sentido específico de éstas fue observar la percepción de la realidad sobre la situación de violencia y comprobar, desde adentro, la visión de los habitantes del país en el período posconflicto, como herencia del enfrentamiento 
o como continuidad de las dinámicas de inseguridad vividas con anterioridad.

Se seleccionaron diversos espacios rurales y urbanos que durante el conflicto fueron escenarios significativos. De igual forma, y como se indicó al principio de este texto, se utilizó la técnica bola de nieve. Para el análisis de datos se utilizó el programa SPSS en su versión 22 (ver gráfica 3).

La violencia, como es bien sabido, no afectó por igual a todas las regiones y grupos del país. El colectivo maya fue el más perjudicado. Se ha de tener en consideración que el genocidio en sí sigue siendo negado por un sector poblacional, por lo que se vio relevante, como punto de partida, comenzar con un cuestionamiento sobre el acontecimiento genocida, que entronca con la violencia pre y posconflicto.

Se ofrecieron tres opciones: "hubo genocidio", "no hubo genocidio" y "no estoy seguro". De los sujetos seleccionados, 187 eran de origen ladino y 15l, mayas. Una minoría, concretamente 38 sujetos, fueron seleccionados entre población extranjera conocedora de la realidad chapina y del proceso diacrónico del país, en especial del conflicto y el genocidio. La elección de un grupo ajeno al enfrentamiento se llevó a cabo con la intencionalidad de reflejar en parte una posición externa, al margen de lo experimentado de manera directa. Las edades de los entrevistados oscilaban entre los 28 y los 74 años. Se observó que, entre la población indígena, una mayoría (77\%) consideró, dos décadas después, que sí existió genocidio. Por otro lado, este hecho contrastó con la opinión del colectivo ladino, en el que sólo el 44\% afirmó que realmente éste tuvo lugar.

\section{¿Cuáles son los problemas fundamentales del país?}

Como se ha indicado, veinte años después persisten la impunidad, las altas tasas de criminalidad y el miedo social. Por tanto, se puede hablar de una continuidad entre la guerra y el período posbélico en determinados indicadores, que se reflejan a su vez en la percepción social de los encuestados (ver gráfica 4). Se puede comprobar que el número de víctimas es incluso superior, de manera proporcional, a los años de la época de enfrentamiento. Existe, como consecuencia, una perseverancia de la criminalidad, con un cambio de agentes, pero con un nivel de generalización destacado.

Posteriormente se realizó una segunda encuesta en los municipios seleccionados sobre las problemáticas que más se detectaban en el día a día, que se amplió a 565 personas, de las cuales el $53.4 \%$ fueron mujeres -del total, 278 eran mayas, y el resto, ladinos o autoindentificados como tales-. Se organizaron las respuestas atendiendo a una escala Likert 0-7. En las respuestas, la preocupación más destacada fue la violencia. Respecto de la pobreza, aunque se consideró una de las cuestiones más significativas, su puntuación resultó fluctuante dependiendo del municipio específico del que se obtuvo la información. La inseguridad fue otra de las mayores problemáticas resaltada por los encuestados, la cual consideraron presente en toda la nación, si bien se observó una diferencia de puntuación relativa entre la ciudad capital y las zonas rurales, donde los homicidios no presentaban un índice tan elevado.

En lo concerniente a la impunidad, a pesar de ser una de las mayores lacras de la sociedad chapina, de manera paradójica no se halló entre las mayores preocupaciones de los entrevistados, al igual que la desigualdad.

\section{¿Ha aumentado la violencia en los últimos años?}

En términos generales, se observó una percepción generalizada de que la violencia había aumentado considerablemente en los últimos años, aunque en la última década no se ha producido un incremento en el número de víctimas. En este sentido, en algunas fuentes, en especial las procedentes de organismos oficiales (INE, ONU) y otras como grupos independientes que buscan la justicia (GAM), la línea de criminalidad resulta bastante plana y, en ocasiones, incluso descendente. Las respuestas pueden tener su origen en un desgaste social por la situación y ausencia de mejoras, debido también a la impunidad y a la crueldad de los crímenes. Este hecho conlleva que la percepción del aumento de la violencia sea mayor (ver gráfica 5). 


\section{Miedo social}

En la observación sistemática llevada a cabo in situ se pudo comprobar un amplio sentimiento de provisionalidad acompañado de fuerte tensión social, lo que conlleva un deterioro de la calidad de vida y un desarraigo social y cultural. Como seindicó previamente, se está produciendo un éxodo de migrantes (ver gráfica 6).

\section{Conclusiones}

Se partió de la hipótesis de que la violencia se halla arraigada en Guatemala como consecuencia de la cultura de la violencia instalada durante más de tres décadas de enfrentamiento. Tras el análisis de los datos y el estudio de campo realizado, se llegó a diversas conclusiones que pueden sintetizarse de la siguiente forma:

1. La violencia perdura, a modo de continuidad, en la época posconflicto. Si se observan los datos de homicidios y las violaciones de los derechos humanos, el número de muertes incrementa cada año de manera proporcional.

2. Siguiendo la estela de la situación actual, hay una cultura de la violencia que se traduce en que la muerte es la principal realidad de la vida. Este hecho se deriva de las altas tasas de criminalidad que se observan en las estadísticas y del miedo social que se comprueba en el análisis de las encuestas realizadas.

3. La violencia se halla generalizada en tres escalas: personal, social y política. Constituye, por tanto, una realidad estructural.

4. La percepción de los colectivos es que los problemas que afectaron al país durante la guerra continúan vigentes en la actualidad. Se percibe escepticismo respecto a las posibles soluciones, como se deriva de las encuestas sobre la mejoría de la situación en los últimos años, así como en la percepción de la baja adecuación de las políticas planteadas.

5. Si se reflexiona sobre el estado de la cuestión y la acuciante realidad que impera, puede concluirse que el fin de la impunidad, del patriarcado excluyente y del etnocentrismo marcado, junto con una reducción de la desigualdad y una educación en la cultura de la paz, podrían tornarse en los únicos procedimientos para emprender un cambio de mentalidad.

El lento proceso de la consecución de la paz en Guatemala conllevó la inauguración de una nueva etapa en la que los colectivos más desfavorecidos parecían hallar una salida a la inequidad, las consecuencias del etnocentrismo y el genocidio que padecieron durante parte del enfrentamiento. Este hecho se vio reforzado por la publicación del informe Guatemala nunca más (ODHAG, 1998) y del Informe de la Comisión para el Esclarecimiento Histórico (CEH) en 1999. Sin embargo, tanto la firma de los acuerdos de paz hace veinte años, como el inicio del proceso de recuperación de la sociedad guatemalteca tras el cruento conflicto, se vieron mancillados por el asesinato de monseñor Gerardi 48 horas después de la publicación del informe de la ODHGA. Se produjo una verdadera inauguración de un periodo de impunidad que en gran medida perdura hasta la actualidad, y que no supone sino la perseverancia de una situación preexistente durante décadas.

Como se ha demostrado en estas páginas, y por los datos ofrecidos, se puede subrayar que existe una cultura de la violencia instalada en Guatemala como eco del enfrentamiento que asoló el país durante casi cuarenta años, y que dos décadas después de su fin sigue dejando un número importante de muertos por la violencia, superior en proporción a los que se registraron en la guerra interna. Esta violencia afecta a todas las capas de la sociedad pero, en especial, a los sectores más desfavorecidos y débiles.

Aunque determinados colectivos están tomando conciencia y desde las bases sociales se esté procediendo a un lento cambio de mentalidad, la violencia tiene un coste humano, social y económico demasiado alto, lo que provoca preocupación y un nuevo éxodo de migrantes que buscan huir de una situación insostenible. 


\section{Referencias}

Aguirre Tobón, Katherine (2014). "Analizando la violencia después del conflicto: el caso de Guatemala en un estudio sub-nacional". En Revista Mexicana de Ciencias Politicas y Sociales, 59(220): 191-233.

Banco Mundial (2010). Crimen y violencia en Centroamérica, vol. II. Guatemala: Banco Mundial.

Bastos, Santiago y Manuela Camus (2003). El movimiento maya en perspectiva. Guatemala: FLACSO.

Benítez Jiménez, María Ixchel (2015). "Frente al genocidio: justicia transicional y movilización en Guatemala". En URVIO. Revista Latinoamericana de Estudios de Seguridad, 16: 37-52.

Cabrera, Luisa (2001). "Efectos de la impunidad en el sentido de la justicia”. En Psicología Política, 23: 37-58.

Cardenal, Ana Sofía (2008). "Maras y pandillas juveniles: dos mundos diferentes. ¿Son las guerras civiles responsables del crimen en Centroamérica?" En Revista CIDOB d'Afers Internacionals, 81: 67-90.

Casaús Arzú, María Elena (2002). La metamorfosis del racismo en Guatemala. Guatemala: Fundación Cholsamaj.

Cea D’Ancona, María de los Ángeles (2001). Metodología cuantitativa. Madrid: Síntesis.

CEH (Comisión para el Esclarecimiento Histórico) (1999). Memoria del silencio. Guatemala: CEH.

CIDH (Comisión Interamericana de Derechos Humanos) (2015). Situación de los derechos humanos en Guatemala: Diversidad, desigualdad y exclusión. Guatemala: CIDH.

Cruz, José Miguel (2010). "Estado y violencia criminal en América Latina”. En Nueva Sociedad, 226: 67-84.

Cuesta Marín, Antonio (2001). Guatemala: la utopía de la justicia. Madrid: Rebelión.

DEMI (Defensoría de la Mujer Indígena) (2007). El acceso de las mujeres indígenas al sistema de justicia oficial de Guatemala. Ukab'wuj ke ixoqib'. Segundo informe. Guatemala: Maya Na'oj.

Duque, Vilma(2007). "Devíctimasdel conflictoapromotores de cambio: trabajo psicosocial y reconciliación en Guatemala". En Revista Futuros, 19(5): 1-16.

Eguizábal, Cristina (2015). Logros y limitaciones de la Comisión Internacional contra la Impunidad en Guatemala
(CICIG). San Salvador: Fundación Nacional para el Desarrollo.

Fernández García, María Cristina (2004). Lynching in Guatemala Legacy of War and Impunity. Cambridge, MA: University of Harvard.

Fernández Rupérez, Emma (2007). "Conflictividad y desafíos actuales en Centroamérica". En CITpax, 6 .

GAM (Grupo de Apoyo Mutuo) (2009). Informe sobre la situación de derechos humanos y hechos de violencia 2009. Guatemala: GAM.

GAM (Grupo de Apoyo Mutuo) (2015a). Informe sobre la situación de derechos humanos y hechos de violencia al mes de marzo 2015. Guatemala: GAM.

GAM (Grupo de Apoyo Mutuo) (2015b). Informe sobre la situación de derechos humanos. Guatemala: GAM.

GAM (Grupo de Apoyo Mutuo) (2016). Informe sobre la situación de derechos humanos. Guatemala: GAM.

GGM (Grupo Guatemalteco de Mujeres) (2016). Estadística de mujeres atendidas por primera vezy seguimiento en la Red de Caimus Periodo enero 2016 al 31 de agosto 2016. Guatemala: GGM

García, Prudencio (2005). El genocidio de Guatemala a la luz de la sociología militar. Madrid: Sepha.

García Carrera, Norma de los Ángeles (2015). "Guatemala, ¿un dolor que debería resignarse?/Guatemala, a Pain That Should Be Resigned?" En Direito da Cidade, 7(1): 251-274.

Gómez Johnson, Cristina (2015). "De la migración económica a la migración forzada por el incremento de la violencia en El Salvador y México". En Estudios Políticos, 47: 199-220.

González, Mariano (2007). "Efectos psicosociales de la violencia en Guatemala”. En Liberación, Revista Digital. 3.

González Sanz, Gonzalo (2014). "Inmunidad política e impunidad en el reconocimiento de genocidio en Guatemala". En Crítica, 989: 8-11.

Gutiérrez, Edgar (1998). "Guatemala. Paz de baja intensidad". En Nueva Sociedad, 157: 1-16.

IARNA (Instituto de Agricultura, Recursos y Ambiente) (2009). Análisis del cumplimiento del objetivo de desarrollo del Milenio relativo a la sostenibilidad del medioambiente en Guatemala y determinación de acciones y costos para alcanzar las metas al año 2015. Guatemala: Universidad Rafael Landívar, SEGEPLAN. 
INACIF (Instituto Nacional de Ciencias Forenses) (2015). Memoria de labores. Guatemala: INACIF.

CIA Word Factbook (2016). Index Mundi. Guatemala. Tasa de mortalidad infantil. Disponible en: http://www. indexmundi.com/es/guatemala/tasa_de_mortalidad infantil.html.

INE (Instituto Nacional de Estadística) (2015). Encuesta Nacional de Condiciones de Vida (ENCOVI). Guatemala: INE.

INE (Instituto Nacional de Estadística) (2016). Estadísticas Vitales. Guatemala: INE.

International Crisis Group (2010). Guatemala: asfixiada entre el crimen y la impunidad. Informe sobre América Latina, 33. Bogotá, Bruselas: ICG.

Ixcol Mus, Carmelita Faustina (2013). El significado de los llamados "linchamientos" en Guatemala y las prácticas que los dotan de sentido. Tesis de doctorado, Universidad Autónoma de Barcelona, Departamento de Psicología Social, Barcelona.

Kalyvas, Stathēs N. (2006) The Logic of Violence in Civil Wars. Nueva York: Cambridge

Kristinsdóttir, Ragna Sigríđur (2015). "Cultura de violencia: normalización de la violencia de género en Guatemala". En Edmé Domínguez y Sádia Castro (eds.), Memorias y movilizaciones de género en América Latina. Gotemburgo: School of Global Studies Universidad de Gotemburgo.

Kurtenbach, Sabine (2008). Youth Violence as a Scapegoat. Youth in Post-War Guatemala. Social and Political Fractures after Wars. Project Working Paper, 5. Duisburg: INEF.

Kurtenbach, Sabine y Herbert Wulf (2012). Violence and Security Concerns in Post-Conflict Situations. Project Working Paper, 3. Duisburg: INEF, Duisburg Essen Universitat

Lambach, Daniel (2007). Oligopolies of Violence in Post Conflict Societies. Working Paper, 62. Leibniz: German Institute of Global and Area Studies.

Maldonado Polanco, Claudia (2009). La incidencia de la cooperación internacional en el proyecto de prevención y erradicación del trabajo infantil en el basurero de la zona 3 de Guatemala. Guatemala: Universidad San Carlos.

McNeish, John-Andrew y Óscar López Rivera (2013). "The Multiple Forms of the Violence in Post-War
Guatemala”. En Astri Suhrke y Mats Berdal (eds.). The Peace in Between: Post-War Violence and Peacebuilding. Reino Unido: Routledge.

Mendoza, Carlos (2003). "Violencia colectiva en Guatemala: una aproximación teórica al problema de los linchamientos". En Carlos Mendoza y Edelberto Torres-Rivas (eds.), Linchamientos: ibarbarie o "justicia popular". Guatemala: FLACSO, pp. 89-104

MINUGUA (Misión de Verificación de las Naciones Unidas en Guatemala) (2001). Los linchamientos: un flagelo contra la dignidad humana. Guatemala: MINUGUA.

OACNUDH (Oficina del Alto Comisionado de las Naciones Unidas para Guatemala) (2016). Informe sobre los Derechos Humanos en Guatemala. Guatemala: OACNUDH.

ODHAG (Oficina de Derechos Humanos del Arzobispado de Guatemala) (1998). Guatemala: nunca más. Informe del Proyecto Interdiocesano de Recuperación de la Memoria Histórica (REHMI). Guatemala: OGHAG.

OMS y OPS (Organización Mundial de la Salud y Organización Panamericana de la Salud) (2016). Perfil de los pueblos indígenas de Guatemala. Guatemala: OMS, Ministerio de Salud Pública.

ONU (Organización de las Naciones Unidas) (2016). Informe de los objetivos del desarrollo sostenible 2016. Nueva York: ONU.

Pásara, Luis (2003). "Acuerdos de paz y cambio en Guatemala”. En Nueva Sociedad, 183: 38-51.

Peacock, Susan C. y Adriana Beltrán (2003). Hidden Powers in Post-Conflict Guatemala: Illegal Armed Groups and the Forces behind Them. Washington: WOLA.

POLJUVE (Políticas Públicas para Prevenir la Violencia Juvenil) (2007). Violencia juvenil, maras y pandillas en Guatemala. Guatemala: POLJUVE, Interpeace.

Ramírez, William (2000). "Derechos humanos en Guatemala. Evidencias de impunidad o impunidad de evidencia”. En Nueva Sociedad, 161:145-158.

Rodríguez Rodríguez, J. y L.M. Gutiérrez Ramírez (2015). "Legados de impunidad y rostros de la verdad en Guatemala. Reflexiones en torno al juicio por genocidio (caso Ríos Montt)". En Revista del Instituto Interamericano de Derechos Humanos, 61(1): 57-86. 
Saavedra, Boris (2014). "La seguridad privada en Guatemala: el caso de la supervisión y el control". En OPERA, 15(15): 55-83.

Sánchez, Susana, Kinnon Scott y Humberto López (2016). Guatemala: cerrando brechas para generar un crecimiento más inclusivo. Diagnóstico sistemático del país. Washington: BM, BIRF.

Sichar Moreno, Gonzalo (2001). Masacres en Guatemala. Guatemala: GAM.

Szichman, Mario (2013). "Reseña de Vivir en el maldito trópico, de David Unger, Plaza y Janés México, 2004". En Revista Universidad de Antioquia, 312: 120-121.

UNICEF (Oficina Regional para América Latina y el Caribe) (2010). Niños y niñas migrantes en situación migratoria irregularen América Latinay el Caribe. Estándares jurídicos básicos y líneas de acción para su protección. Buenos Aires: UNICEF.

UNICEF (Fondo de las Naciones Unidas para la Infancia) (2014). El estado mundial de la infancia. Todos los niños y niñas cuentan. Revelando las imparidades para impulsar los derechos de la niñez. Nueva York: UNICEF.

UNICEF (Fondo de las Naciones Unidas para la Infancia) (2015). Principales avances y desafíos del Estado y el pueblo de Guatemala en el cumplimiento de los derechos de la infancia con motivo de los 25 años de la Convención sobre los Derechos del Niño. Guatemala: UNICEF.

UNICEF (Fondo de las Naciones Unidas para la Infancia) (2016). Estado mundial de la infancia. Una oportunidad para cada niño. Nueva York: UNICEF.
UNDP (United Nations Development Programme) (2011). Human Development Report 2011. Sustainability and Equity: A Better Future for All. Nueva York: UNDP. UNDP (United Nations Development Programme) (2016). Human Development Report 2016. Nueva York: UNDP.

Valdez, Julio (2016). "Paradojas de la inclusión de género y etnia en Guatemala". En Análisis de la Realidad Nacional, 100: 15.

Varela Huerta, Amarela (2016). "El Movimiento Migrante Mesoamericano. Una aproximación desde la sociología de la acción colectiva a un ejemplo de luchas migrantes". En Amnis. Revue de Civilisation Contemporaine Europes/Amériques, 15: 1-10.

Vela Castañeda, Manolo Estuardo (2014). Los pelotones de la muerte: la construcción de los perpetradores del genocidio Guatemalteco. México: El Colegio de México.

Vilas, Carlos M. (2005). "Linchamiento: venganza, castigo e injusticia en escenarios de inseguridad". En El Cotidiano, 131: 20-26.

Villa, Juan David, Daniela Londoño Díaz y Daniela Barrera Machado (2015). "Reparación a las víctimas de dictaduras, conflictos armados y violencia política en sus componentes de compensación, satisfacción, rehabilitación y no repetición”. En El Ágora USB, 15(1): 217-240.

Zartman, William (1995). Elusive Peace: Negotiating an End to Civil Wars. Washington D.C.: The Brookings Institution.

Zuzunaga Ruiz, Roger (2011). "La peligrosa expansión de los Zetas”. En Boletín Electrónico del IDEI, 40: 2-4. 
Gráfica 1. Homicidios en Guatemala 2000-2015

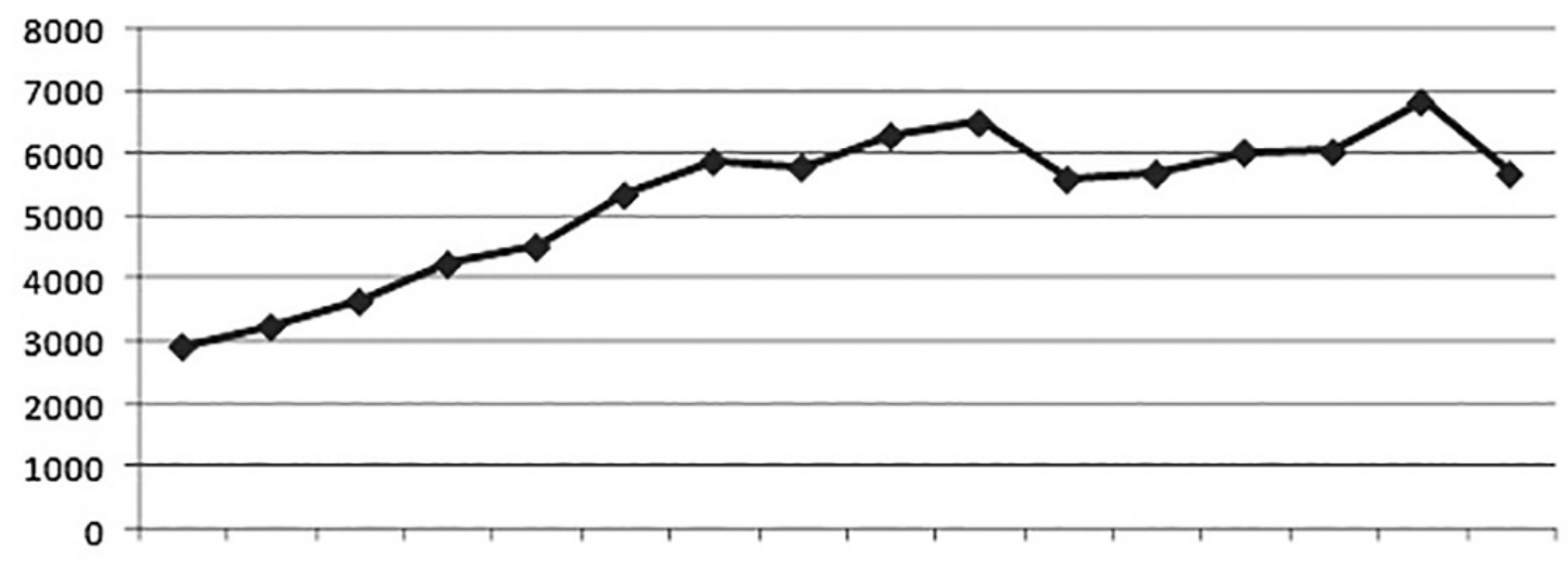

2000200120022003200420052006200720082009201020112012201320142015

Fuente: elaboración propia con base en información proporcionada por el GAM (2015b y 2016); INE (2016), el Grupo Guatemalteco de Mujeres (GGM, 2016) y el Instituto Nacional de Ciencias Forenses (INACIF, 2015).

Gráfica 2. Linchamientos 2000-2016

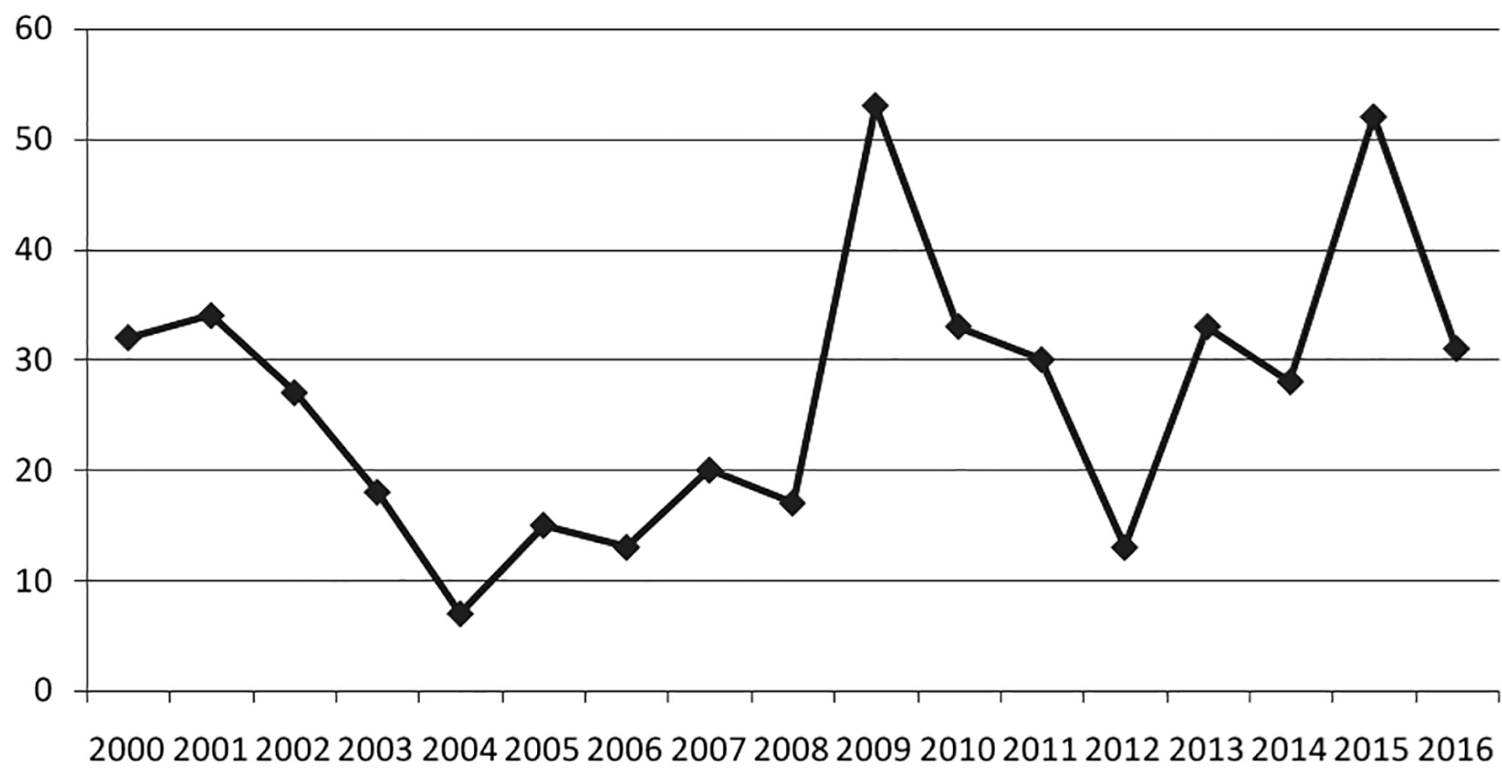

Fuente: elaboración propia con base en GAM (2009, 2015 y 2016), MINUGUA (2001), CIDH (2015) e INE (2016). 
Gráfica 3. ¿Hubo genocidio?

-Hubo genocidio

No hubo genocidio
No estoy seguro

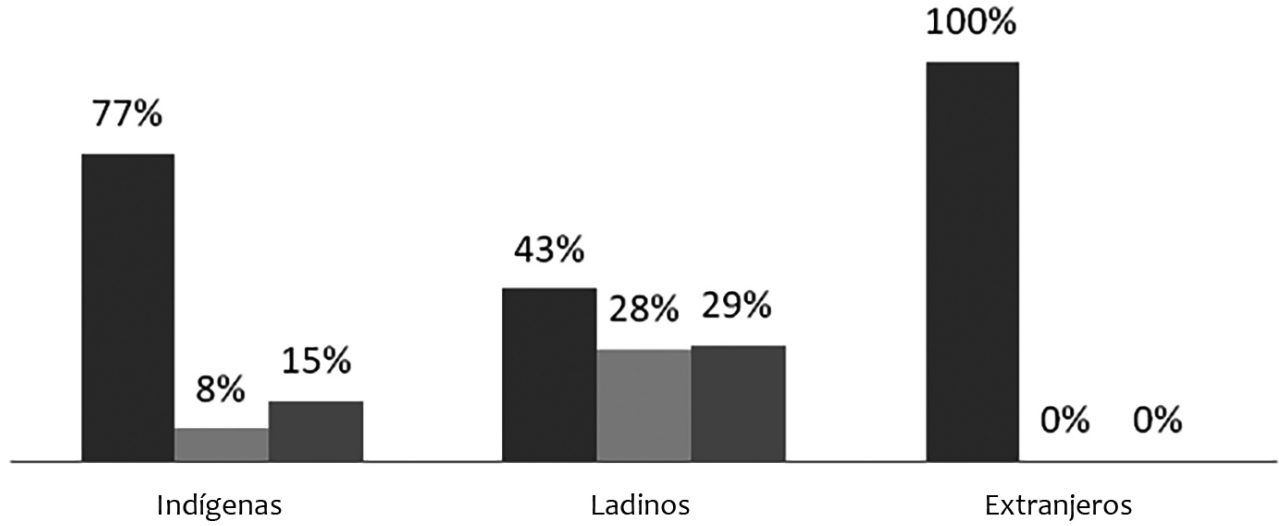

Fuente: elaboración propia con base en encuestas.

Gráfica 4. Principales problemas en el país

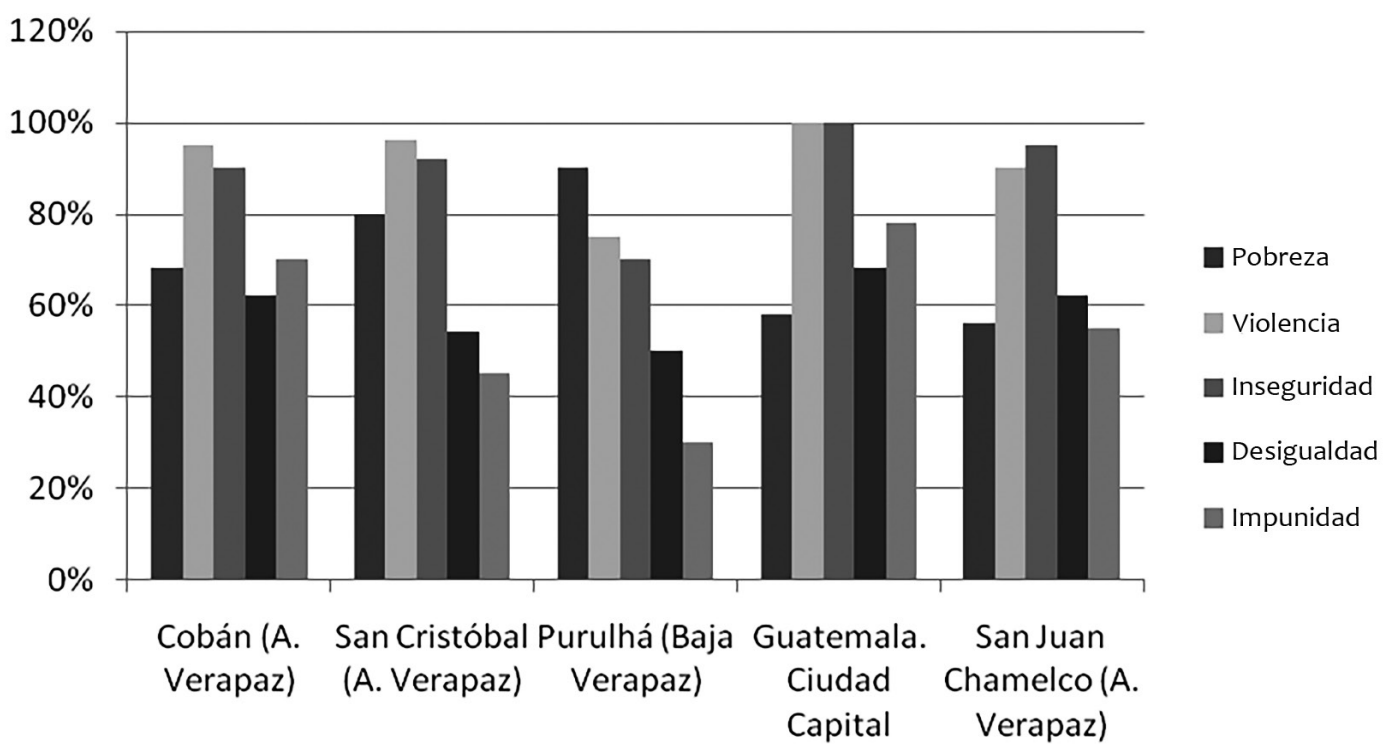

Fuente: elaboración propia con base en encuestas. 
Gráfica 5. Situación de la violencia en los últimos años. ¿Se ha agravado la violencia en el periodo 2011-2015?

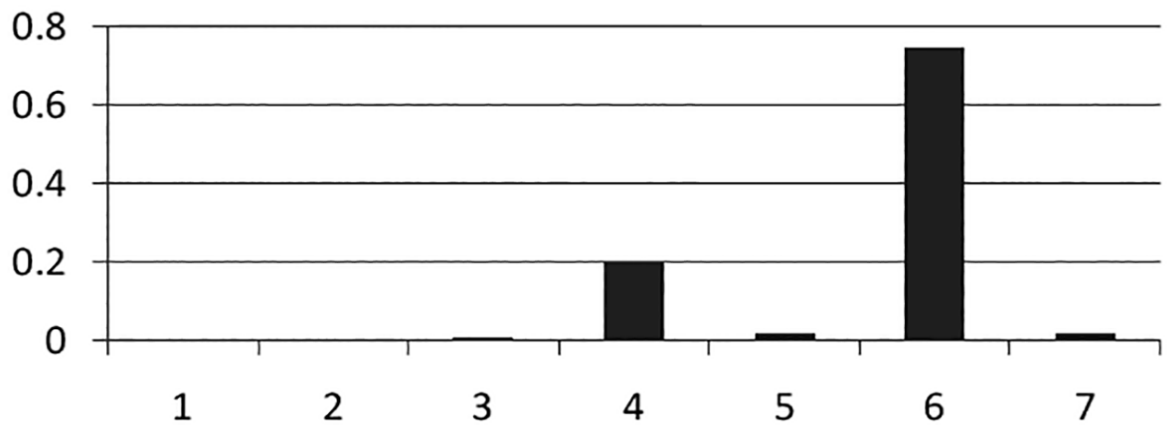

Fuente: elaboración propia con base en encuestas.

Nota: la gráfica refleja las opiniones extraídas de la escala Likert de la encuesta, en la que 1 implica el máximo desacuerdo y 7 el máximo acuerdo.

Gráfica 6. ¿Son adecuadas las políticas contra la violencia?

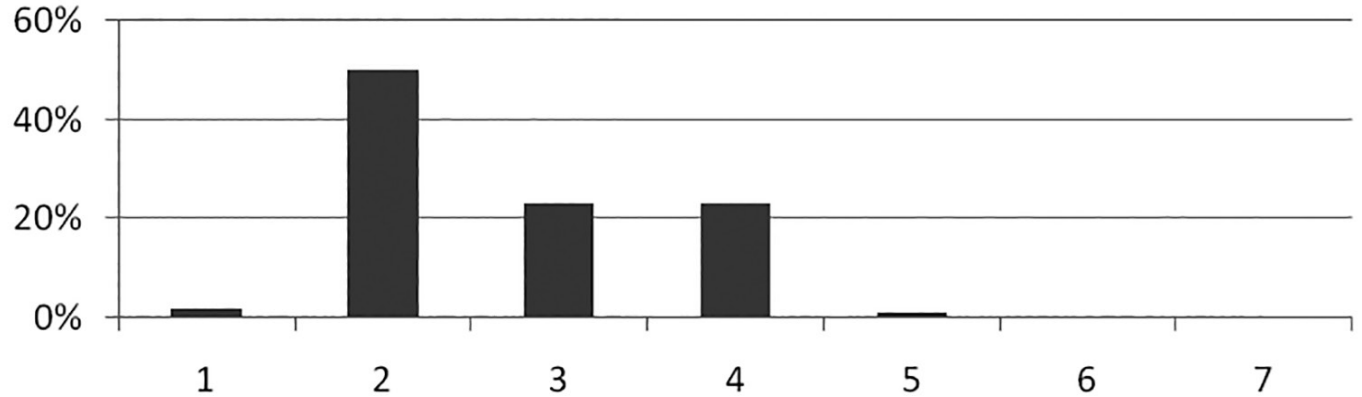

Fuente: elaboración propia con base en encuestas.

Nota: la gráfica refleja las opiniones extraídas de la escala Likert de la encuesta, en la que 1 implica el máximo desacuerdo y 7 el máximo acuerdo. 
Gráfica 7. ¿Existe miedo social?

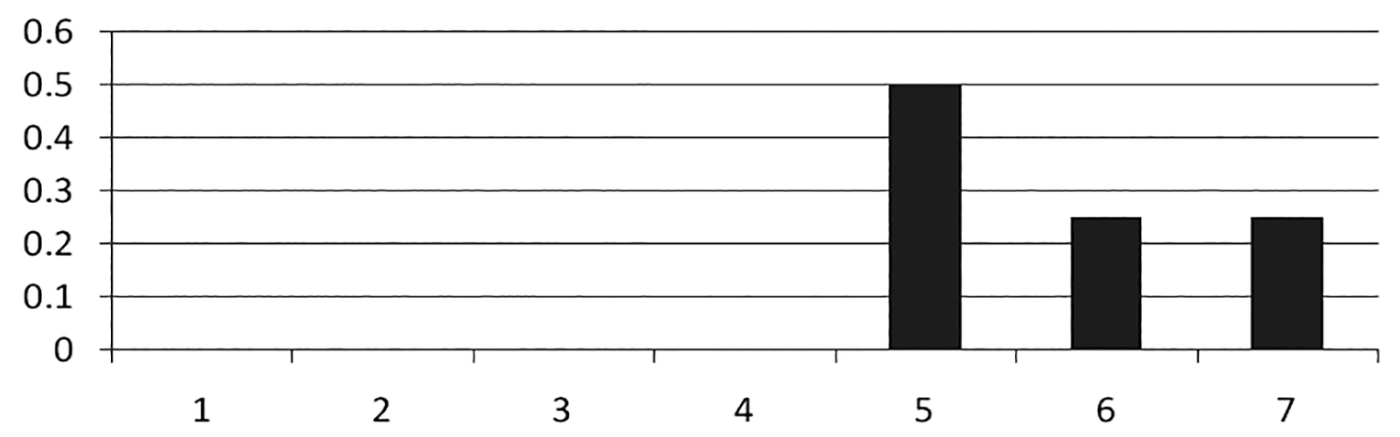

Fuente: elaboración propia con base en encuestas.

Nota: la gráfica refleja las opiniones extraídas de la escala Likert de la encuesta, en la que 1 implica el máximo desacuerdo y 7 el máximo acuerdo.

Tabla 1. Tasa de criminalidad y número de muertes violentas en el periodo 2008-2016

\begin{tabular}{cccc}
\hline Año & Tasa de criminalidad & $\begin{array}{c}\text { Número de víctimas } \\
\text { violentas según el GAM }\end{array}$ & $\begin{array}{c}\text { Número de víctimas } \\
\text { violentas según la } \\
\text { policía y fuentes } \\
\text { oficiales }\end{array}$ \\
\hline $\mathbf{2 0 0 8}$ & 46.00 & 6292 & 6302 \\
$\mathbf{2 0 0 9}$ & 46.36 & 6498 & 6498 \\
$\mathbf{2 0 1 0}$ & 41.50 & 5590 & 5960 \\
$\mathbf{2 0 1 1}$ & 38.61 & 5681 & 5681 \\
$\mathbf{2 0 1 3}$ & 34.20 & 6024 & $5155^{*}$ \\
2014 & 34.02 & 6032 & $5253^{*}$ \\
2016 & 31.62 & $635 / 5875$ & $4998 *$ \\
\hline
\end{tabular}

Fuente: elaboración propia con base en información proporcionada por el en GAM (2009, 2015 y 2016), MINUGUA (2001), CIDH (2015) e INE (2016).

* Las cifras manifiestan la discrepancia significativa entre los datos ofrecidos por las fuentes oficiales y por el GAM. 
Tabla 2. Evolución de la mortalidad infantil 2000-2014

\begin{tabular}{ll}
\hline Año & Tasa \\
\hline 2000 & 47.03 \\
2001 & 45.79 \\
2002 & 44.55 \\
2003 & 37.92 \\
2004 & 36.91 \\
2005 & 35.93 \\
2006 & 30.94 \\
2007 & 29.77 \\
2008 & 28.79 \\
2009 & 27.84 \\
2010 & 26.91 \\
2011 & 26.02 \\
2012 & 25.16 \\
2013 & 24.32 \\
2014 & 23.51 \\
\hline & \\
\hline &
\end{tabular}

Fuente: elaboración propia con base en Data CIAWORLD Factbook (2016), IARNA (2009) y UNICEF (2014, 2015). 